\title{
EFFICIENT ONE-POT SYNTHESIS OF BENZOXAZOLE DERIVATIVES CATALYZED BY NICKEL SUPPORTED SILICA
}

\author{
SURESH MADDILA*, SREEKANTH. B JONNALAGADDA \\ School of Chemistry, University of KwaZulu-Natal, West Ville Campus, Chilten Hills, Private Bag 54001, \\ Durban-4000, South Africa \\ (Received: May 27, 2011 - Accepted: March 29, 2012)
}

\begin{abstract}
A simple and efficient method has been developed for the synthesis of benzoxazoles from 2-aminophenol and substituted aldehydes in the presence of a catalytic amount of nickel supported silica at room temperature.
\end{abstract}

Keywords: Nickel suppoted silica, Benzoxazoles, One-pot two-component synthesis, 2-Aminophenol, Substituted aldehydes.

\section{INTRODUCTION}

The benzoxazole moiety is the key structure feature of a large number of biologically active natural products and pharmaceutical compounds ${ }^{1}$. Two protocols for the synthesis of benzoxazoles have been developed. One is the copper-catalyzed intramolecular $o$-arylations or intermolecular domino annulations of $o$-arylhalides ${ }^{2-4}$ and the other is the direct condensation of 2-aminophenol with carboxylic acid or aldehyde under harsh conditions, such as in the presence of strong acid, high temperature ${ }^{5}$ or strong oxidants ${ }^{6-9}$. Catalytic aerobic oxidation using oxygen as terminal oxidant has received much attention ${ }^{10,11}$ and been used in the synthesis of benzoxazoles ${ }^{12,13}$.

Nickel supported silica as an environmental friendly and economical catalyst has been attracting increasing research interest from chemists ${ }^{14}$. Although kinds of $\mathrm{Ni}$ supported $\mathrm{SiO}_{2}$ catalyzed organic transformations have been developed, the $\mathrm{Ni}-\mathrm{SiO}_{2}$ to form carbon-carbon and carbon-heteroatom bond has remained largely undeveloped ${ }^{15}$. Herein, we report an efficient and environmentally friendly method for the synthesis of benzoxazoles catalyzed by nickel supported silica at room temperature (Scheme 1).

We studied the possibility to synthesis of benzoxazoles by the reaction of 2-aminophenol and substituted aldehyde using $\mathrm{Ni}-\mathrm{SiO}_{2}$ as the catalyst (Scheme 1). Here, an efficient and simple method for the synthesis of target compounds is described and the synthesis of some compounds has been reported in our previous studies.

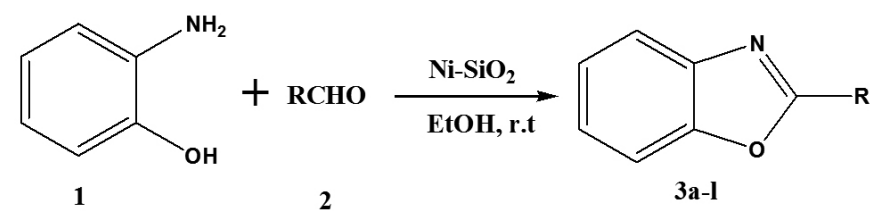

Scheme 1

\section{EXPERIMENTAL SECTION}

All reagents and solvents were purchased and used without further purification. Crude products were purified by column chromatography on silica gel of 60-120 mesh. ${ }^{1} \mathrm{H}$ and ${ }^{13} \mathrm{C}$ NMR spectra were recorded on the $400 \mathrm{MHz}$ instruments, and spectral data are reported in ppm relative to tetramethylsilane (TMS) as internal standard. LCMS Mass spectra were recorded on a MASPEC low resolution mass spectrometer operating at $70 \mathrm{eV}$.

To conclude, we have shown that the Ni supported silica is a highly active catalyst for the synthesis of benzoxazoles. General procedure for the preparation of benzoxazoles: A mixture of 2-aminophenol $(1.5 \mathrm{mmol})$ and substituted aldehydes $(1 \mathrm{mmol})$ with $\mathrm{Ni}$ supported $\mathrm{SiO}_{2}(20 \mathrm{~mol} \%)$ in $\mathrm{EtOH}$ $(10 \mathrm{~mL})$ was stirred at ambient temperature for an appropriate time (Table 1). After completion of the reaction as indicated by TLC, the $\mathrm{Ni}_{-} \mathrm{SiO}_{2}$ was filtered and washed with $50 \% \mathrm{EtOH}(2 \times 10 \mathrm{~mL})$. The crude product was purified by recrystallization from diethyl ether (solid products) or by chromatography using silica gel and mixtures of hexane/ethyl acetate of increasing polarity. The physical data was identified by ${ }^{1} \mathrm{H}$ NMR, ${ }^{13} \mathrm{C}$ NMR and LCMS spectrometers.

\section{RESULTS AND DISCUSSION}

2-aminophenol and substituted aldehydes were selected as the model reaction to examine catalytic activity of $\mathrm{Ni}$ supported $\mathrm{SiO}_{2}$ at ambient temperature. To indicate the need of $\mathrm{Ni}$ supported $\mathrm{SiO}_{2}$ for this condensation. We observed that the model reaction did not proceed in the absence of $\mathrm{SiO}_{2}$ even after $24 \mathrm{~h}$ (Table 1, entry 1). When using catalytic amount of $10 \mathrm{~mol} \% \mathrm{Ni}^{2}$ $\mathrm{SiO}_{2}$, the reaction gave benzoxazoles with $70 \%$ yield in $1.5 \mathrm{~h}$ in EtOH (Table 1 , entry 3), and further lowering the catalyst loading up to $5 \mathrm{~mol} \%$ led to lower yield of $55 \%$ in $1.5 \mathrm{~h}$ (Table 1, entry 2). In the presence of $20 \mathrm{~mol} \%$ catalyst the reaction affords the corresponding synthesis of benzoxazoles in $98 \%$ yield within $1.5 \mathrm{~h}$ (Table 1 , entry 4$)$, and $\mathrm{Ni}-\mathrm{SiO}_{2}(25 \mathrm{~mol} \%$ ) also gives $98 \%$ yield in $1.5 \mathrm{~h}$ (Table 1 , entry 5 ). The solvents examined were trichloromethane, acetonitrile and ethanol, among which ethanol is shown to be the best (Table 1). Accordingly, $20 \mathrm{~mol} \% \mathrm{Ni}-\mathrm{SiO}_{2}$ catalysts loading in $\mathrm{EtOH}$ is considered optimal for the synthesis of benzoxazoles.

Table 1: Two-component synthesis of benzoxazoles under various conditions $s^{\mathrm{a}}$.

\begin{tabular}{|c|c|c|c|}
\hline Entry & Catalyst (\%) & Time (hour) & Yield $^{\mathrm{b}}(\%)$ \\
\hline 1 & No catalyst & $24 \mathrm{~h}$ & 0 \\
\hline 2 & 5 & $1.5 \mathrm{~h}$ & 55 \\
\hline 3 & 10 & $1.5 \mathrm{~h}$ & 70 \\
\hline 4 & 20 & $1.5 \mathrm{~h}$ & 98 \\
\hline 5 & 25 & $1.5 \mathrm{~h}$ & 98 \\
\hline 6 & 20 & $2 \mathrm{~h}$ & $86^{\mathrm{c}}$ \\
\hline 7 & 20 & $2.5 \mathrm{~h}$ & $90^{\mathrm{d}}$ \\
\hline
\end{tabular}

a $\mathrm{Ni}-\mathrm{SiO}_{2}$ was added to a mixture of $1.5 \mathrm{mmol}$ of 2-aminophenol and 1 mmol of aromatic aldehydes.

${ }^{\mathrm{b}}$ Isolated yield.

${ }^{c}$ In the presence of $\mathrm{CHCl}_{3}$.

${ }^{\mathrm{d}} \mathrm{In}$ the presence of $\mathrm{CH}_{3} \mathrm{CN}$.

To word, we prepared a range of benzoxazoles under the optimized conditions (Table 2). 2-Aminophenol, different aldehydes were coupled with under these reaction conditions. The reactions are clean and highly selective affording exclusively benzoxazoles in high yields in a short reaction time. The reaction of 2-aminophenol coupled with 3,4-dimethyl, 4-methyl and 4-methoxy is completed within $1.5 \mathrm{~h}$ with $98 \%, 96 \%$ and $94 \%$ yield, respectively (Table 2 , entries 3a-3c). Similar reaction of 2-aminophenol coupled with simple benzaldehyde produces the corresponding products in excellent yield of $93 \%$ in $1.5 \mathrm{~h}$, respectively (Table 2, entry $\mathbf{3 d}$ ). This method is equally effective with electron-withdrawing 4-fluoro, 4-trifluoromethyl and 4-chloro benzaldehydes produces the corresponding products in $89 \%, 86 \%$ and $88 \%$ yield in 'longer' 
reaction time $2.5,3$ and $2.5 \mathrm{~h}$ in respectively (Table 2 , entries $\mathbf{3 e - 3 g}$ ). The reaction of 2-aminophenol coupled with 3-thienyl, 4-pyridyl and 1-naphthyl benzaldehyde produces the corresponding products in $91 \%, 93 \%$ and $90 \%$ yield in $1.5 \mathrm{~h}$, respectively (Table 2, entries $\mathbf{3 h}-\mathbf{3 j}$ ). The reaction of 2-aminophenol coupled with ethyl, butylaldehyde produces the corresponding products in $94 \%$, and $94 \%$ yield in $1.5 \mathrm{~h}$, respectively (Table 2 , entries $\mathbf{3 k} \mathbf{k} \mathbf{3 l}$ ).

Table 2: Preparation of various benzoxazoles in the presence of $\mathrm{Ni}_{-} \mathrm{SiO}_{2}$ in $\mathrm{EtOH}$ at room temperature .

\begin{tabular}{|c|c|c|c|}
\hline Entry & $\mathrm{R}$ & Time (hour) & Yield (\%) \\
\hline $3 a$ & $3,4-\mathrm{MeC}_{6} \mathrm{H}_{3}$ & 1.5 & 98 \\
\hline $3 b$ & $4-\mathrm{MeC}_{6} \mathrm{H}_{4}$ & 1.5 & 96 \\
\hline $3 c$ & $4-\mathrm{MeOC}_{6} \mathrm{H}_{4}$ & 1.5 & 94 \\
\hline 3d & $\mathrm{H}$ & 1.5 & 93 \\
\hline $3 e$ & $4-\mathrm{FC}_{6} \mathrm{H}_{4}$ & 2.5 & 89 \\
\hline $3 f$ & 4- $\mathrm{CF}_{3} \mathrm{C}_{6} \mathrm{H}_{4}$ & 3.0 & 86 \\
\hline $3 g$ & $4-\mathrm{ClC}_{6} \mathrm{H}_{4}$ & 2.5 & 88 \\
\hline $3 \mathrm{~h}$ & 3-thienyl & 1.5 & 91 \\
\hline $3 \mathbf{i}$ & 4-pyridyl & 1.5 & 93 \\
\hline $\begin{array}{l}3 \mathbf{j} \\
3 \mathbf{k} \\
3 \mathbf{l}\end{array}$ & $\begin{array}{c}\text { 1-naphthyl } \\
\text { ethyl } \\
\text { butyl }\end{array}$ & $\begin{array}{l}1.5 \\
1.5 \\
1.5\end{array}$ & $\begin{array}{l}90 \\
94 \\
94\end{array}$ \\
\hline
\end{tabular}

${ }^{a}$ Reaction conditions: 2-aminophenol (1.5 mmol), substituted aldehyde (1 $\mathrm{mmol}), \mathrm{Ni}_{-} \mathrm{SiO}_{2}(20 \mathrm{~mol} \%$ ), room temperature, $\mathrm{EtOH}$.

2-(3,4-Dimethylphenyl)benzoxazole (3a): ${ }^{1} \mathrm{H}$ NMR $\left(\mathrm{CDCl}_{3}, 400 \mathrm{MHz}\right.$, ppm): $\delta 8.10(\mathrm{~d}, J=8.1 \mathrm{~Hz}, 2 \mathrm{H}, \mathrm{Ar}-\mathrm{H}), 7.75(\mathrm{t}, J=7.6 \mathrm{~Hz}, 1 \mathrm{H}, \mathrm{Ar}-\mathrm{H}), 7.57(\mathrm{t}$, $J=7.5 \mathrm{~Hz}, 1 \mathrm{H}, \mathrm{Ar}-\mathrm{H}), 7.36-7.24(\mathrm{~m}, 3 \mathrm{H}, \mathrm{Ar}-\mathrm{H}), 2.40\left(\mathrm{~s}, 6 \mathrm{H}, \mathrm{CH}_{3}\right) ;{ }^{13} \mathrm{C} \mathrm{NMR}$ $\left(\mathrm{CDCl}_{3}, 100 \mathrm{MHz}, \mathrm{ppm}\right): \delta 163.0,150.2,141.6,140.9,132.5,130.9,128.4$, $125.7,124.4,124.6,121.6,119.6,110.4,20.1,18.9$; MS (70 eV, EI): $m / z(\%)$ : $224(\mathrm{M}+1)$.

2-P-tolylbenzoxazole (3b): ${ }^{1} \mathrm{H}$ NMR $\left(\mathrm{CDCl}_{3}, 400 \mathrm{MHz}, \mathrm{ppm}\right): \delta 8.15(\mathrm{~d}$, $J=8 \mathrm{~Hz}, 2 \mathrm{H}, \mathrm{Ar}-\mathrm{H}), 7.79(\mathrm{t}, J=7.7 \mathrm{~Hz}, 1 \mathrm{H}, \mathrm{Ar}-\mathrm{H}), 7.57(\mathrm{t}, J=7.6 \mathrm{~Hz}, 1 \mathrm{H}$, $\mathrm{Ar}-\mathrm{H}), 7.36-7.30(\mathrm{~m}, 4 \mathrm{H}, \mathrm{Ar}-\mathrm{H}), 2.42\left(\mathrm{~s}, 3 \mathrm{H}, \mathrm{CH}_{3}\right) ;{ }^{13} \mathrm{C} \mathrm{NMR}\left(\mathrm{CDCl}_{3}, 100\right.$ MHz, ppm): $\delta 163.2,150.6,142.1,141.9,129.5,127.5,124.8,124.4,124.3$ 119.8, 110.4, 21.5; MS (70 eV, EI): $m / z(\%): 209\left(\mathrm{M}^{+}\right)$.

2-(4-Methoxyphenyl)benzoxazole (3c): ${ }^{1} \mathrm{H}$ NMR $\left(\mathrm{CDCl}_{3}, 400 \mathrm{MHz}\right.$, ppm): $\delta 8.20$ (d, $J=9.2 \mathrm{~Hz}, 2 \mathrm{H}, \mathrm{Ar}-\mathrm{H}), 7.76$ (t, $J=7.8 \mathrm{~Hz}, 1 \mathrm{H}, \mathrm{Ar}-\mathrm{H}), 7.56$ $(\mathrm{t}, J=7.5 \mathrm{~Hz}, 1 \mathrm{H}, \mathrm{Ar}-\mathrm{H}), 7.36-7.29(\mathrm{~m}, 2 \mathrm{H}, \mathrm{Ar}-\mathrm{H}), 7.03(\mathrm{~d}, J=8.8 \mathrm{~Hz}, 2 \mathrm{H}$ $\mathrm{Ar}-\mathrm{H}), 3.88$ (s, $\left.3 \mathrm{H}, \mathrm{OCH}_{3}\right) ;{ }^{13} \mathrm{C}$ NMR $\left(\mathrm{CDCl}_{3}, 100 \mathrm{MHz}, \mathrm{ppm}\right): \delta 163.1,162.3$, 150.6, 142.2, 129.3, 124.6, 124.4, 119.7, 119.6, 114.3, 110.3, 55.4; MS (70 eV, EI): $m / z(\%): 226(\mathrm{M}+1)$.

2-Phenylbenzoxazole (3d): ${ }^{1} \mathrm{H}$ NMR $\left(\mathrm{CDCl}_{3}, 400 \mathrm{MHz}, \mathrm{ppm}\right): \delta 8.29$ $8.26(\mathrm{~m}, 2 \mathrm{H}, \mathrm{Ar}-\mathrm{H}), 7.81(\mathrm{t}, J=7.7 \mathrm{~Hz}, 1 \mathrm{H}, \mathrm{Ar}-\mathrm{H}), 7.60-7.51(\mathrm{~m}, 4 \mathrm{H}, \mathrm{Ar}-\mathrm{H})$ 7.38-7.34 (m, 2H, Ar-H); ${ }^{13} \mathrm{C}$ NMR $\left(\mathrm{CDCl}_{3}, 100 \mathrm{MHz}, \mathrm{ppm}\right): \delta 162.9,150.7$, $142.0,131.4,128.8,127.5,127.1,125.0,124.5,119.9,110.5 ; \mathrm{MS}(70 \mathrm{eV}, \mathrm{EI})$ $m / z(\%): 196(\mathrm{M}+1)$.

2-(4-Fluorophenyl)benzoxazole (3e): ${ }^{1} \mathrm{H} N \mathrm{NM}\left(\mathrm{CDCl}_{3}, 400 \mathrm{MHz}, \mathrm{ppm}\right)$ : $\delta 8.26(\mathrm{~d}, J=8.2,2 \mathrm{H}, \mathrm{Ar}-\mathrm{H}), 7.78(\mathrm{t}, J=7.9 \mathrm{~Hz}, 1 \mathrm{H}, \mathrm{Ar}-\mathrm{H}), 7.57(\mathrm{t}, J=7.4$ $\mathrm{Hz}, 1 \mathrm{H}, \mathrm{Ar}-\mathrm{H}), 7.36(\mathrm{~d}, J=8.1 \mathrm{~Hz}, 2 \mathrm{H}, \mathrm{Ar}-\mathrm{H}), 7.21(\mathrm{~d}, J=7.9 \mathrm{~Hz}, 2 \mathrm{H}, \mathrm{Ar}-\mathrm{H})$ ${ }^{13} \mathrm{C}$ NMR $\left(\mathrm{CDCl}_{3}, 100 \mathrm{MHz}, \mathrm{ppm}\right): \delta 164.7,162.0,150.7,141.9,129.8,129.7$, 125.0, 124.6, 123.4, 119.9, 116.1, 115.9, 110.5; MS (70 eV, EI): $\mathrm{m} / \mathrm{z}(\%): 214$ $(\mathrm{M}+1)$.

2-(4-(Trifluoromethyl)phenyl)benzoxazole (3f): ${ }^{1} \mathrm{H}$ NMR $\left(\mathrm{CDCl}_{3}, 400\right.$ $\mathrm{MHz}, \mathrm{ppm}): \delta 8.55$ (d, $J=8.3 \mathrm{~Hz}, 1 \mathrm{H}, \mathrm{Ar}-\mathrm{H}), 8.45(\mathrm{~d}, J=8.2 \mathrm{~Hz}, 1 \mathrm{H}, \mathrm{Ar}-$ $\mathrm{H}), 7.83(\mathrm{t}, J=7.8 \mathrm{~Hz}, 2 \mathrm{H}, \mathrm{Ar}-\mathrm{H}), 7.69-7.59(\mathrm{~m}, 2 \mathrm{H}, \mathrm{Ar}-\mathrm{H}), 7.44-7.37(\mathrm{~m}$, $2 \mathrm{H}, \mathrm{Ar}-\mathrm{H}) ;{ }^{13} \mathrm{C}$ NMR $\left(\mathrm{CDCl}_{2}, 100 \mathrm{MHz}, \mathrm{ppm}\right): \delta 161.5,150.8,141.9,131.8$, 130.6, 129.5, 128.0, 127.9, 125.7, 124.9, $124.5(J=4 \mathrm{~Hz}), 120.3$, 110.7; MS (70 eV, EI): $m / z(\%): 263(\mathrm{M}+1)$.

2-(4-(Chlorophenyl)benzoxazole (3g): ${ }^{1} \mathrm{H}$ NMR $\left(\mathrm{CDCl}_{3}, 400 \mathrm{MHz}\right.$, $\mathrm{ppm}): \delta 8.17(\mathrm{~d}, J=8.1 \mathrm{~Hz}, 2 \mathrm{H}, \mathrm{Ar}-\mathrm{H}), 7.79(\mathrm{t}, J=7.5 \mathrm{~Hz}, 1 \mathrm{H}, \mathrm{Ar}-\mathrm{H}), 7.54(\mathrm{t}$, $J=7.2 \mathrm{~Hz}, 1 \mathrm{H}, \mathrm{Ar}-\mathrm{H}), 7.49(\mathrm{~d}, J=7.3 \mathrm{~Hz}, 2 \mathrm{H}, \mathrm{Ar}-\mathrm{H}), 7.37(\mathrm{~d}, J=7.4 \mathrm{~Hz}, 2 \mathrm{H}$ $\mathrm{Ar}-\mathrm{H}) ;{ }^{13} \mathrm{C} \mathrm{NMR}\left(\mathrm{CDCl}_{3}, 100 \mathrm{MHz}, \mathrm{ppm}\right) . \delta 161.9,150.7,141.9,137.6,129.2$, 128.7, 125.6, 125.2, 124.6, 120.0, 110.5; MS (70 eV, EI): $\mathrm{m} / z(\%): 230(\mathrm{M}+1)$

2-(Thiophen-3-yl)benzoxazole (3h): ${ }^{1} \mathrm{H}$ NMR $\left(\mathrm{CDCl}_{3}, 400 \mathrm{MHz}, \mathrm{ppm}\right)$ : $\delta 8.20(\mathrm{dd}, J=2.8,1.0 \mathrm{~Hz}, 1 \mathrm{H}), 7.80(\mathrm{dd}, J=4.8,1.0 \mathrm{~Hz}, 1 \mathrm{H}), 7.78(\mathrm{t}, J=7.8$
$\mathrm{Hz}, 1 \mathrm{H}, \mathrm{Ar}-\mathrm{H}), 7.58(\mathrm{t}, J=7.4 \mathrm{~Hz}, 1 \mathrm{H}, \mathrm{Ar}-\mathrm{H}), 7.44$ (s, 1H, Ar-H), 7.37-7.32 (m, 2H, Ar-H); ${ }^{13} \mathrm{C}$ NMR $\left(\mathrm{CDCl}_{3}, 100 \mathrm{MHz}, \mathrm{ppm}\right): \delta 159.7,150.3,141.9$, $129.2,128.0,126.9,126.6,124.9,124.5,119.9,110.4$; MS (70 eV, EI): $m / z$ (\%): $201\left(\mathrm{M}^{+}\right)$.

2-(Pyridine-4-yl)benzoxazole (3i): ${ }^{1} \mathrm{H} \mathrm{NMR}\left(\mathrm{CDCl}_{3}, 400 \mathrm{MHz}, \mathrm{ppm}\right): \delta$ $8.79(\mathrm{~d}, J=7.9 \mathrm{~Hz}, 2 \mathrm{H}), 8.05(\mathrm{~d}, J=8.1 \mathrm{~Hz}, 2 \mathrm{H}, \mathrm{Ar}-\mathrm{H}), 7.81(\mathrm{t}, J=7.5 \mathrm{~Hz}$, $1 \mathrm{H}, \mathrm{Ar}-\mathrm{H}), 7.62(\mathrm{t}, J=7.3 \mathrm{~Hz}, 1 \mathrm{H}, \mathrm{Ar}-\mathrm{H}), 7.42-7.35(\mathrm{~m}, 2 \mathrm{H}) ;{ }^{13} \mathrm{C} \mathrm{NMR}$ $\left(\mathrm{CDCl}_{3}, 100 \mathrm{MHz}, \mathrm{ppm}\right): \delta 160.5,150.7,150.6,141.6,134.2,126.2,125.0$, 120.9, 120.6, 110.8; MS (70 eV, EI): $m / z$ (\%): 197 (M+1).

2-(Naphthalene-1-yl)benzoxazole (3j): ' $\mathrm{H}$ NMR $\left(\mathrm{CDCl}_{3}, 400 \mathrm{MHz}\right.$, ppm): $\delta 9.54$ (d, $J=8.4 \mathrm{~Hz}, 1 \mathrm{H}), 8.46(\mathrm{~d}, \mathrm{~J}=8.2 \mathrm{~Hz}, 1 \mathrm{H}, \mathrm{Ar}-\mathrm{H}), 8.03$ (d, $J=8.4$ $\mathrm{Hz}, 1 \mathrm{H}, \mathrm{Ar}-\mathrm{H}), 7.97(\mathrm{t}, \mathrm{J}=7.7 \mathrm{~Hz}, 2 \mathrm{H}, \mathrm{Ar}-\mathrm{H}), 7.76(\mathrm{t}, \mathrm{J}=7.2 \mathrm{~Hz}, 1 \mathrm{H}, \mathrm{Ar}-\mathrm{H})$, 7.69-7.58 (m, 3H, Ar-H), 7.45-7.44 (m, 2H, Ar-H); ${ }^{13} \mathrm{C} \mathrm{NMR}\left(\mathrm{CDCl}_{3}, 100\right.$ MHz, ppm): $\delta 162.7,150.1,142.3,133.9,132.2,130.6,129.2,128.6,127.8$, $126.4,126.3,125.2,124.8,124.4,123.5,120.2,110.4 ; \mathrm{MS}(70 \mathrm{eV}, \mathrm{EI}): \mathrm{m} / z$ (\%): $246(\mathrm{M}+1)$.

2-Ethylbenzoxazole (3k): ${ }^{1} \mathrm{H}$ NMR $\left(\mathrm{CDCl}_{3}, 400 \mathrm{MHz}, \mathrm{ppm}\right): \delta 7.60(\mathrm{~d}, \mathrm{~J}$ $=7.6 \mathrm{~Hz}, 1 \mathrm{H}, \mathrm{Ar}-\mathrm{H}), 7.42(\mathrm{~d}, \mathrm{~J}=7.2 \mathrm{~Hz}, 1 \mathrm{H}, \mathrm{Ar}-\mathrm{H}), 7.23(\mathrm{t}, \mathrm{J}=8.3 \mathrm{~Hz}, 2 \mathrm{H}$, $\mathrm{Ar}-\mathrm{H}), 2.93-2.85\left(\mathrm{q}, J=7.3 \mathrm{~Hz}, 2 \mathrm{H}, \mathrm{CH}_{2}\right), 1.40-1.19\left(\mathrm{t}, J=7.63 \mathrm{~Hz}, 3 \mathrm{H}, \mathrm{CH}_{3}\right)$; ${ }^{13} \mathrm{C}$ NMR $\left(\mathrm{CDCl}_{3}, 100 \mathrm{MHz}, \mathrm{ppm}\right): \delta 167.4,150.9,141.5,124.6,124.2,119.7$, 110.4, 22.4, 11.1; MS (70 eV, EI): $m / z(\%): 148(\mathrm{M}+1)$.

2-Butylbenzoxazole (3I): ${ }^{1} \mathrm{H}$ NMR (CDCl $\left., 400 \mathrm{MHz}, \mathrm{ppm}\right): \delta 7.67(\mathrm{~d}, \mathrm{~J}$ $=7.8 \mathrm{~Hz}, 1 \mathrm{H}, \mathrm{Ar}-\mathrm{H}), 7.47(\mathrm{~d}, \mathrm{~J}=7.5 \mathrm{~Hz}, 1 \mathrm{H}, \mathrm{Ar}-\mathrm{H}), 7.29(\mathrm{t}, \mathrm{J}=8.3 \mathrm{~Hz}, 2 \mathrm{H}$, $\mathrm{Ar}-\mathrm{H}), 2.93\left(\mathrm{t}, J=7.6 \mathrm{~Hz}, 2 \mathrm{H}, \mathrm{CH}_{2}\right) 1.89-1.81\left(\mathrm{~m}, 2 \mathrm{H}, \mathrm{CH}_{2}\right), 1.46-1.40(\mathrm{~m}$, $\left.2 \mathrm{H}, \mathrm{CH}_{2}\right), 0.97\left(\mathrm{t}, J=7.6 \mathrm{~Hz}, 3 \mathrm{H}, \mathrm{CH}_{3}\right) ;{ }^{13} \mathrm{C} \mathrm{NMR}\left(\mathrm{CDCl}_{3}, 100 \mathrm{MHz}\right): \delta 167.6$, $150.9,141.6,124.6,124.2,119.7,110.5,29.0,28.6,22.5,13.9$; MS $(70 \mathrm{eV}$, $\mathrm{EI}): \mathrm{m} / z(\%): 176(\mathrm{M}+1)$.

\section{CONCLUSION}

In conclusion, we have developed a novel and highly efficient method for the synthesis of benzoxazoles by treatment of 2-aminophenol and substituted aldehyde in the presence of $\mathrm{Ni}$ supported silica as an effective Lewis acid. The significant advantages of this methodology are good yields, short reaction times, a simple workup procedure, and easy preparation and handling of the catalyst. This methodology may find widespread uses in organic synthesis for preparation of the benzoxazoles.

\section{ACKNOWLEDGEMENTS}

The authors are thankful to the authorities of School of Chemistry, University of KwaZulu-Natal, Westville campus, Durban, South Africa for the facilities and encouragement.

\section{REFERENCES}

1. R. D. Viirre, G. Evindar, R. A. Batey, Journal of Organic Chemistry, 73, $3452,(2008)$.

2. G. Altenhoff, F. Glorius, Advanced Synthesis \& Catalysis, 346, 1661, (2004).

3. G. Evindar, R. A. Batey, Journal of Organic Chemistry, 71, 1802, (2006).

4. N. Barbero, M. Carril, R. SanMartin, E. Domínguez, Tetrahedron, 63, 10425, (2007)

5. M. Terashima, M. Ishii, Y. Kanaoka, Synthesis, 484, (1982).

6. K. H. Park, K. Jun, S. R. Shin, S. W. Oh, Tetrahedron Letters, 37, 8869, (1996).

7. R. S. Varma, R. K. Saini, O. Prakash, Tetrahedron Letters, 38, 2621, (1997).

8. J. Chang, K. Zhao, S. Pan, Tetrahedron Letters, 43, 951, (2002).

9. C. Praveen, K. H. Kumar, D. Muralidharan, P. T. Perumal, Tetrahedron, 64, 2369, (2008).

10. G. J. Ten Brink, I. W. C. E. Arends, R. A. Sheldon, Chemical Reviews, 104, 4105, (2004).

11. M. Beller, Advanced Synthesis \& Catalysis, 346, 107, (2004).

12. Y. Kawashita, N. Nakamichi, H. Kawabata, M. Hayashi, Organic Letters, 5, 3713, (2003).

13. Y. X. Chen, L. F. Qian, W. Zhang, B. Han, Angewandte Chemie International Edition, 47, 9330, (2008).

14. A. Rahman, S. B. Jonnalagadda, Catalysis Letters, 123, 264, (2008).

15. A. Rahman, V. S. R. R. Pullabhotla, S. B. Jonnalagadda, Catalysis Communications, 9, 2417, (2008). 